\title{
Using Artificial Bees Colony Algorithm for License Plate Recognition
}

\author{
Yu Weiyu ${ }^{\mathrm{a}}$, Hu Dan ${ }^{\mathrm{b}}$, Li Chuyi ${ }^{\mathrm{c}}$ \\ School of Electronic and Information Engineering, South China University of Technology, \\ Guangzhou, P. R. China 510641 \\ ayuweiyu@scut.edu.cn, b254232612@qq.com, 419895133@qq.com
}

Keywords: vehicle plate recognition, artificial bee colony algorithm, image preprocessing

\begin{abstract}
In this paper, a new vehicle plate recognition method is proposed using the artificial bee colony algorithm. This method can be implemented in entrance admission, security, airport or harbor cargo control, traffic control. Artificial bee colony algorithm was first introduced by Dervis Karaboga in 2005 for solving numerical optimization problems. ABC algorithm has achieved better results on the optimization problems. We use ABCA on image transformation, enhancement, denoise and BP network. Experimental results showed proposed method is effective and effect.
\end{abstract}

\section{Introduction}

Vehicle plate recognition can be used in many applications such as entrance admission, security, airport or harbor cargo control, traffic control. It also can improve the traffic efficiency, enhance vehicle identification degree, reduce artificial management, parking lot systems. A lot of researches focus on this area and it is the need of the hour for a better secured society and for an efficient monitoring of road rules [1-3]. Vehicle plate follows a process of motion detection followed by character segmentation and finally character detection to extract the characters from a vehicle plate. There are many constrains in extracting the vehicle plate such as illumination conditions, noise, uneven shadowing, non uniform size of the license plate text. At night, tracking of the vehicles is a challenging task, includes false noise pixels which are misunderstood for motion detection. Proposed method can be divided into two methods: (i) plate preprocessing from captured images using artificial bee colony algorithm and (ii) recognition of character of the vehicular plate using BP network.

\section{Artificial Bee Colony Algorithm}

Artificial bee colony algorithm (ABCA) was first introduced by Karaboga in 2005 for solving numerical optimization problems [4]. In ABCA, the bee colony is divided into three categories: employed bees, onlooker bees and scouts.

(1) Employed bees: All bees that are currently exploiting a food source are classified as 'employed'. There is only one employed bee for every food source, i.e., the number of food sources equals to the number of employed bees. They share the nectar information of the food sources with the onlookers.

(2) Onlooker: "Onlookers"' are those bees that are waiting in the hive for the information to be shared by the employed bees about their food sources.

(3) Scouts: "Scouts"' are those bees which are currently searching for new food sources in the vicinity of the hive.

The steps of the algorithm as follows:

Initialize the population of bee colony. Set all the bees as the scouts.

Assess the effectiveness of each nectar according to the value of the objective function.

Each employed bee searches for new nectar in respective areas in accordance with the Equ. (1).

$$
V_{i j}=X_{i j}+R_{i j}\left(X_{i j}-X_{k j}\right)
$$


Here, $V_{i j}$ represents the new location of nectar. $X_{i j}$ represents the j-dimensional position of the i-th nectar. $X_{k j}$ represents the j-dimensional position of the k-th nectar $(k \neq i) . R_{i j}$ is a random number between -1 to 1 .

Determine whether to update the original nectar based on greedy selection strategy.

An onlooker bee evaluates the nectar information taken from all employed bees and chooses a food source with a probability related to its nectar amount. This probabilistic selection is really a roulette wheel selection mechanism which is described as Equ.(2)

$$
\mathrm{P}_{i}=\frac{f i t_{i}}{\sum_{j} f i t_{j}}
$$

Where $f i t_{i}$ represents the fitness value of the $i_{t h}$ solution in the swarm. As seen, the better the solution $\mathrm{i}$, the higher the probability of the $i_{t h}$ food source selected. Each onlooker search for new nectar in the selected areas and determine whether to update the original nectar based on greedy selection strategy.

If a position cannot be improved over a predefined number of cycles, then the food source is abandoned. Assume that the abandoned source is $X_{i}$, and then the scout bee discovers a new food source to be replaced with $i_{t h}$ as shown in Equ(3):

$$
X_{i k}=1 b_{j}+\operatorname{rand}(0,1) \times\left|u b_{j}-1 b_{j}\right|
$$

Where rand $(0,1)$ is a random number within $[0,1]$ based on a normal distribution. $1 b, u b$ are lower and upper boundaries of the $i^{\text {th }}$ dimension respectively.

Record the optional solution at this iteration. Repeat the above steps until the end of the cycle.

\section{Vehicle Plate Location}

The flow diagram of vehicle plate recognition is shown in Figure1.

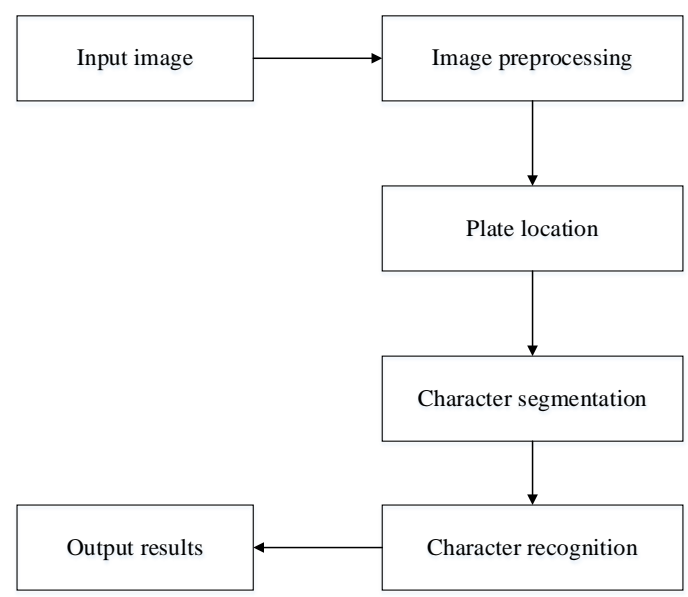

Figure1 flow chart of vehicle plate recongnition

we need to find the location of the license plate, the steps are as follows:

1) transform the input image to gray image;

2) gray level transformation;

3) median filtering is performed to remove impulse noise or salt-pepper noise;

4) use open and close operation to remove small regions;

5) color license plate is extracted from some candidate region according to the color and size of the license plate. 
In this section, vehicle plate need to preprocess in order to recognition character better.

\section{Enhancement}

A license plate area is accounted for $20 \%$ of the whole license plate area. We know the license plate characters and background is very difference due to other reasons, but the overall character of pixel values higher than the background pixel values are also. So we can enhancement license plate area of the top $20 \%$ of pixels for other pixels are suppressed, thus to enhance the purpose of target character suppress background.

Here, we use Incomplete Beta function [5], shown in Equ.(4):

$$
F(u)=\frac{1}{\int_{0}^{1} t^{\alpha-1}(1-t)^{\beta-1} d_{t}} \times \int_{0}^{1} \int_{0}^{1} t^{\alpha-1}(1-t)^{\beta-1} d_{t}
$$

Here, $\alpha, \beta$ is variable. We choose different values for different license plate image. The steps is as follows:

1) normalize the image of license plate, as shown in Equ.(5)

$$
f^{\prime}(x, y)=\left[f(x, y)-L_{\min }\right] /\left(L_{\max }-L_{\min }\right)
$$

Where $\mathrm{f}(\mathrm{x}, \mathrm{y})$ is the original gray value, $\mathrm{f}^{\prime}(\mathrm{x}, \mathrm{y})$ is the normalized gray value, $L_{\min }$ and $L_{\max }$ are the minimum and maximum of the original gray value.

2) Quality of images can be evaluated based on the human vision or PSNR, image sharpness and image contrast. Considering the quality requirements of images, we introduce image contrast C, image information entropy $\mathrm{H}$ and image compactness $\mathrm{Z}$ to measure image quality. We come to the fitness function of ABCA for image enhancement. Shown in Equ.(6):

$$
\text { Fitness }=C \times 10+H+Z / 100
$$

We multiply image contrast $C$ by 10 and divided image compactness $Z$ by 100 . That is to ensure the three indexes in the same order of magnitude, which controls the consistency of the weight of the image quality assessment. Obviously, the larger the value of Fitness, the better the image quality.

\section{Denoise}

In this section, noise is suppressed using artificial bee colony algorithm[6]. Noise has been concentrated in the mark, the pixel value is estimated by the pollution. The optimization objective function is shown as Equ(7).

$$
\left.F_{X}\right|_{N}(u)=\sum_{(i, j) \in N}\left[\left|X_{i j}-u_{i j}\right|\right]+\sum_{(m, n) \in V_{i j} \cap N} \varphi\left(u_{i j}-u_{m n}\right)
$$

Here, $\mathrm{N}$ is the matrix of the coordinates of the noise according to the different method, its size is consistent with the size of the damaged image. $V_{i j}$ presents Neighborhood of $(i, j), \varphi(t)=\sqrt{a+t^{2}}$. Set $\alpha=0.001$.

To minimize the value of $F_{x}$, corresponding to the optimal solution, the corresponding elements of the optimal solution are used to replace the noise pixels in the contaminated image, that is, the image is obtained after the noise reduction.

\section{Character Segmentation}

License plate images and the projection curve of it are shown in figure2. When we are looking for segmentation point of the second character and the third character, we will find two trough points candidate " $A$ " and the point " $D$ ". At the moment, we first regard point " $A$ " as a true point of division, then segment character according to point " $A$ ", detect character position index is in line with the actual characteristics after segment end. If the character sequence segmented is in line with the actual characteristics of the split, segment the first two characters. Otherwise, character is segmented based on the point " $D$ " again. If point " $A$ " and " $D$ " are indivisible in line with the characteristics of license 
plate character sequence, this candidate region represents the as false license plate area, then to discard it.

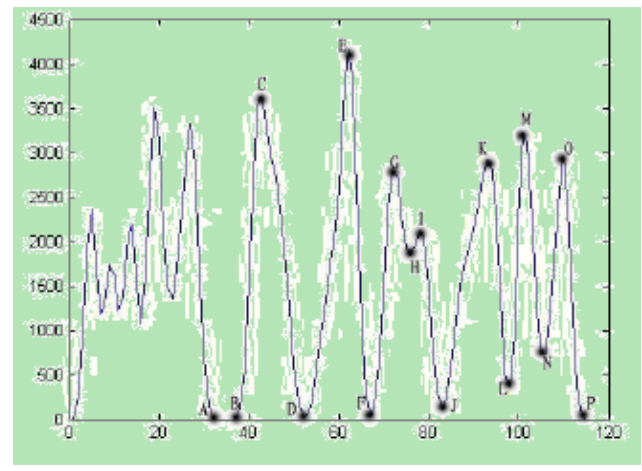

Figure 2 vehicle plate and projection curve

In figure 2, when we get the split point A, we can segment character. First, we need the statistics all the peaks and troughs appear behind the split points. In statistics, we use "three point" method to judge the peaks and troughs, but caused by noise wave which we don't need, so we need to get rid of the peak and the trough points caused by noise, so when we judge by three wave troughs agreed about crest value difference is not too large, otherwise think the trough and the wave caused by noise, we will not be counted. We get the point after processing is needed to do character segmentation, if the split points behind a wave Valley and ordinate is zero, will be reset to dividing point to order. In the diagram above, point A segmentation is followed A wave Valley point B and point B y coordinate is zero, so the set point to point $B$ and then began to character segmentation.

\section{BP Neural Network for recognition}

We use Artificial Bee Colony algorithm during the training process of BP neural network (BPNN). With the initialization of weights and biases in BPNN, ABCA is imported to generate these parameters and optimize them by sufficient quantities of iterations, which can be used in the training of BPNN model in return.

The fitness function in the ABCA can be defined as the loss function in the BPNN model, which we use Mean Square Error (MSE) indeed. Consequently, smaller loss of BPNN implies the better performance of searching in Artificial Bee Colony algorithm.

\section{Experiment results}

The system was implemented in Matlab and tested on real test images. Figure 3 showed image preprocessing. Figure 4 is vehicle plate processing. Figure 5 is character segmentation.

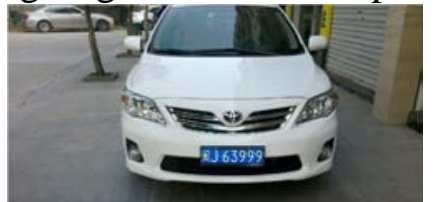

(a) original image

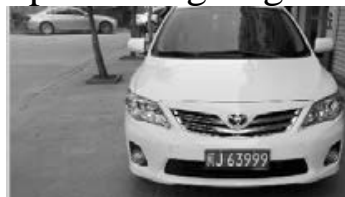

(b) gray image

Figure 3 enhancement image

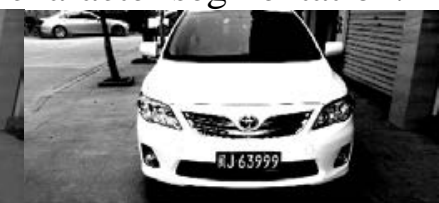

(c)enhancement image

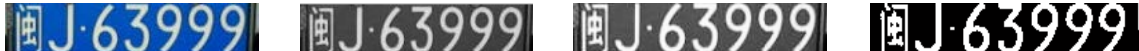

(a)Color image (b)gray image (c) enhancement image (d) binary image

\section{阅了. 63999}

(e)Dilate image

Figure 4 vehicle plate processing

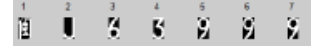

Figure 5 Characters segmentation

\section{Conclusion}


The vehicle plate recognition system is proposed for the detection of the number plates of the vehicles which can be used for further processes like parking, storing, allowing vehicle to pass or reject vehicle. This paper implements ABCA to successfully segment number plate area from the original image, and applies a character recognition to get the vehicle license number. We designed the system using ABCA with improved robustness. Experimental results showed proposed method is effective and effect.

\section{References}

[1] Ching-Liang Su. Car plate recognition by whole 2-D image. Expert Systems with Applications, Vol.38, Iss. 6, 2011, p7195-7200.

[2] Fikriye Öztürk, Figen Özen. A New License Plate Recognition System Based on Probabilistic Neural Networks, Procedia Technology, Vol.1, 2012, p.124-128.

[3] Yu Wang, Xiaojuan Ban, Jie Chen, Bo Hu, Xing Yang. License plate recognition based on SIFT feature.Optik-International Journal for Light and Electron Optics, Vol.126, Iss.21, No. 2015, p.2895-2901.

[4] Karaboga D, Basturk B. A powerful and efficient algorithm for numerical function optimization: artificial bee colony (ABC) algorithm [J]. Journal of global optimization, 2007, 39(3),p.459-471.

[5] Jia Chen, Chu-Yi Li, Wei-Yu Yu. Adaptive image enhancement based on artificial bee colony algorithm. CEIE2016(accept).

[6] Xiao Yonghao, Yu Weiyu, Chen Yongchang, Tian Jing. An effective approach for removing heavy salt-peppers noise based on bee colony optimisation. International Journal of Computational Science and Engineering, vol.6, no 1-2, 2011,p.60-66. 